\title{
Histopathological study on the prevalence of trichosporonosis among autopsy subjects using peptide nucleic acid probe in situ hybridization technique
}

Sota Sadamoto, Megumi Wakayama, Minoru Shinozaki, Yasuhiro Nihonyanagi, Kozue Ejima, Aki Mituda, Naobumi Tochigi, Tetsuo Nemoto, Kazutoshi Shibuya Department of surgical pathology, Toho University, School of Medicine

\section{Summary}

To distinguish Trichosporon spp. from Candida spp. we underwent in situ hybridization (ISH) technique by using peptide nucleic acid (PNA) probe which contain specific nucleotide sequence target of $C$. albicans and Trichosporon spp. Finally, 7 cases of trichosporonosis were confirmed, 5 out of which had been diagnosed as candidiasis.

In conclusion, some Trichosporonosis may be mingled with cases recoded as candidiasis in past. We also wish to emphasize usefulness of ISH applying to histological examination to diagnose invasive fungal infections.

\section{Introduction}

Trichosporon species, which are mostly insusceptible to echinocandins, are some of the most common dimorphic yeasts in Asia including Japan. We sometimes encounter breakthrough trichosporonosis in individuals being treated with echinocandins. Thus, it is therefore important to distinguish between Trichosporon spp. and Candida spp. through histopathological examination. However, the morphological similarity of these fungi makes it difficult to distinguish between these fungi only by routine histological examination. For choosing optimal antifungal drugs, favorable molecular supplemental procedure is required to identify these fungi for histopathological diagnosis. In our previous studies, we developed peptide nucleic probes (PNA) designed to be specific for $C$. albicans and Trichosporon spp. The purpose of this study is to evaluate the utility of an ISH procedure focusing on the histopathological prevalence of trichosporonosis in autopsy subjects by using PNA probe ISH.

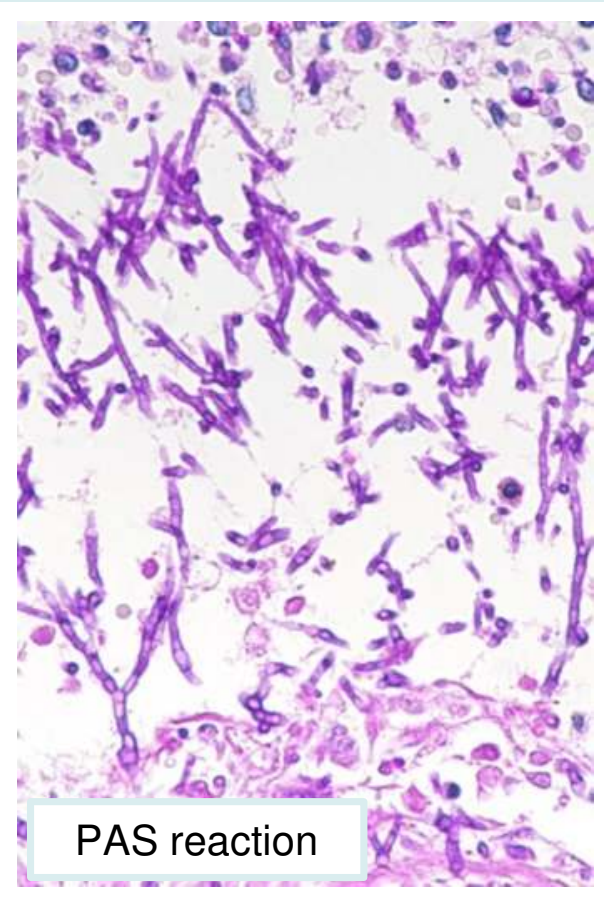

\section{Material and Method}

Formalin-fixed and paraffin embedded (FFPE) tissue samples used in this study were blood stream yeast infection of autopsy. These autopsy cases were corrected from two major regional hospital in Tokyo during 1975-2012 were used in this study. Three expert pathologists took part in

histopathological examination to verify the previous diagnosis of invasive yeast infection and exclude cryptococcosis and mold infections from our cohort. ISH was performed with PNA probes of $C$. albicans and Trichosporon spp.

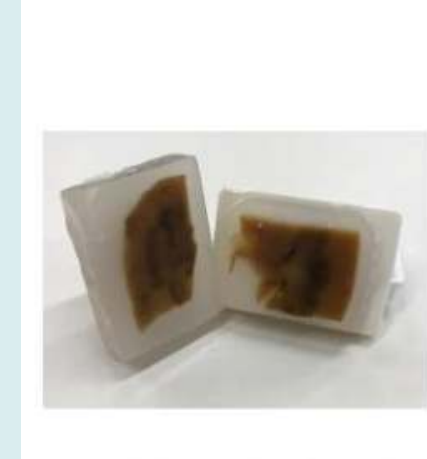

Formalin fixed and paraffin embedded tissue (FFPE)
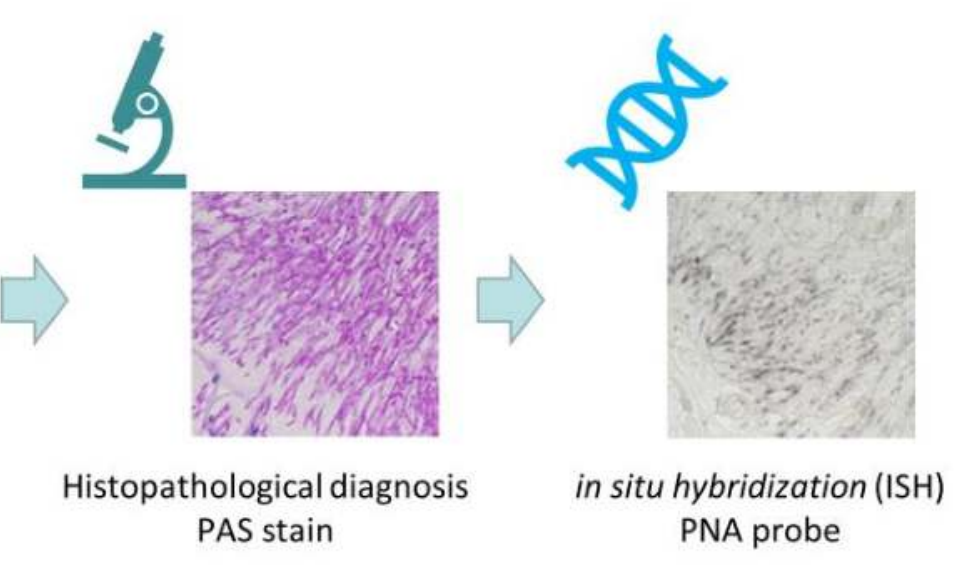

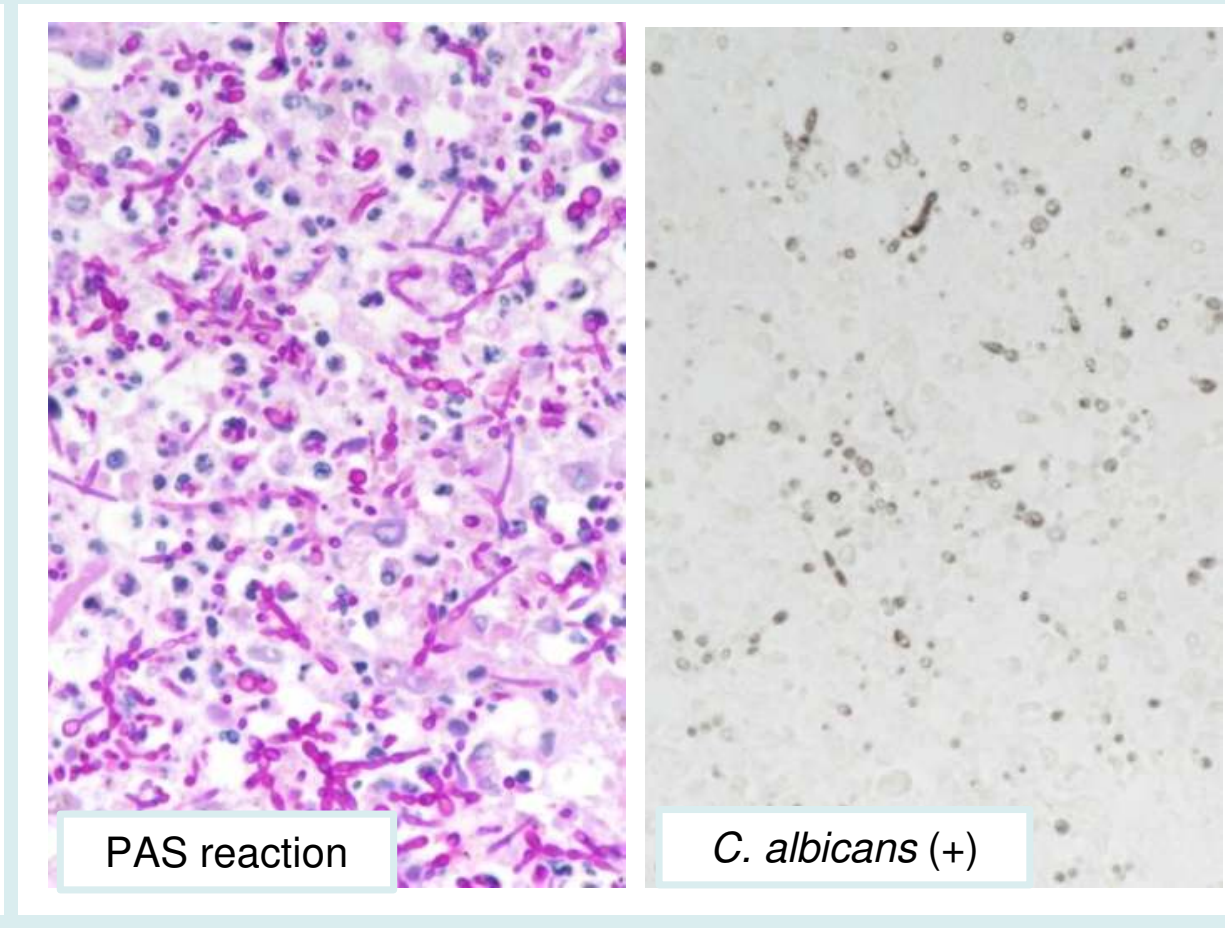

\section{Result}

Figure 1 summarize the outline. 7 cases showed a positive signal for the Trichosporon spp. probe and 29 cases for the C. albicans probe. 5 of the 7 Trichosporon spp. probe positive cases had been diagnosed as candiasis in the past autopsy reports.

Autopsy FFPE specimens with invasive yeast infection 95 cases \begin{tabular}{|l|l|l|}
\hline Histopathological diagnosis & $\longrightarrow \begin{array}{l}\text { Non budding yeast with PAS positive capsule (cryptococcosis) } 5 \text { cases } \\
\text { Mold infection } 2 \text { cases }\end{array}$ \\
\hline
\end{tabular}

Non-cryptococcosis yeast infection 88 cases

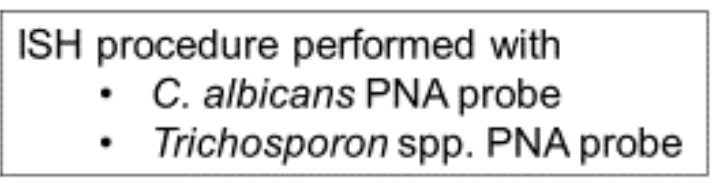

Negative cases to both probe 52 cases

$>$ No target gene inside the yeast

(non-albicans Candida spp. etc.

$>$ The target gene damage

(ex. Formalin, Reaction of immune system of hosts)
Nucleotide sequences of the primers and probes used in the study

Probe target Nucleotide sequence Probe length and labeling
ACAGCAGAAGCCGTG $\begin{gathered}15 \text { mer } \\ \text { PNA }\end{gathered} \quad$ N terminus: FITC ACAGCAGAAGCCTG $\mathrm{P}$,

\section{Conclusion}

The present study identified 7 cases of invasive trichosporonosis, 5 of which had been misdiagnosed as candidiasis. The misdiagnoses indicate that incidence of trichosporonosis may be higher than previously reported. Our results show that ISH is an useful tool to identify Trichosporon spp. in FFPE tissue samples. 